REVIEW ARTICLE

\section{A.A.K. Abdel Razek}

M. Castillo

\title{
Imaging Lesions of the Cavernous Sinus
}

\begin{abstract}
SUMMARY: Our aim was to review the imaging findings of relatively common lesions involving the cavernous sinus (CS), such as neoplastic, inflammatory, and vascular ones. The most common are neurogenic tumors and cavernoma. Tumors of the nasopharynx, skull base, and sphenoid sinus may extend to the CS as can perineural and hematogenous metastases. Inflammatory, infective, and granulomatous lesions show linear or nodular enhancement of the meninges of the CS but often have nonspecific MR imaging features. In many of these cases, involvement elsewhere suggests the diagnosis. MR imaging is sensitive for detecting vascular lesions such as carotid cavernous fistulas, aneurysms, and thromboses.
\end{abstract}

$\mathbf{T}$ he cavernous sinus (CS) contains vital neurovascular structures that may be affected by vascular, neoplastic, infective, and infiltrative lesions arising in the CS proper or via extension from adjacent intra- and extracranial regions. $\mathrm{Pa}-$ tients with CS syndrome usually present with paresis of 1 or more cranial nerves (III-VI), which may be associated with painful ophthalmoplegia. The clinician needs to know the type of CS lesion, its relationship to crucial neurovascular structures, and its extension into the surrounding tissues. These findings are essential for deciding therapeutic modalities such as microsurgery, radiation therapy, or medical treatment as well as for appropriate planning of surgery or radiation therapy. ${ }^{1-8}$ Because the MR imaging features of many of these lesions overlap and are often nonspecific, we will emphasize those features that allow one to formulate a reasonable differential diagnosis.

\section{Imaging Protocol and Anatomy}

MR imaging of the CS should include routine T2, fluid-attenuated inversion recovery (FLAIR), and precontrast T1weighted images of the entire brain. Postcontrast T1-weighted $\leq 3$-mm-thick images should be obtained in the axial and coronal planes with at least 1 plane imaged with a fat-saturation technique. Thin-section postcontrast axial images may be acquired by $3 \mathrm{D}$ spoiled gradient techniques. We generally image from the orbital apex through the prepontine cistern. Thin-section 3D heavily T2-weighted images (such as constructive interference in steady state or fast imaging employing steady-state acquisition) may allow visualization of individual cranial nerves in the CS and adjacent cisterns. CT is best performed by using a multidetector scanner after intravenous administration of iodinated contrast medium. Acquisitions in axial or coronal planes by using $<1$-mm-thick sections may be obtained and then reformatted in other planes. ${ }^{2-7}$

The CS is composed of 2 layers of dura that split to form a septate venous channel. Each dural wall contains an outer layer apposed to bone and an inner layer in contact with blood or CSF. The CS extends from the orbital apex and superior

Received August 14, 2008; accepted after revision October 2.

From the Department of Diagnostic Radiology (A.A.K.A.R.), Masnoura Faculty of Medicine, Mansoura, Egypt; and Department of Radiology (M.C.), University of North Carolina, Chapel Hill, NC.

Please address correspondence to Ahmed Abdel Khalek Abdel Razek, MD, Diagnostic Radiology Department, 62 El Nokri St, Meet Hadr, Mansoura Faculty of Medicine, Mansoura, Egypt; e-mail: arazek@mans.eun.eg

Indicates open access to non-subscribers at www.ajnr.org

DOI 10.3174/ajnr.A1398 orbital fissure anteriorly to the Meckel cave and farther posteriorly to the dura and the pores that allow nerves to enter it. Its transverse diameter is $5-7 \mathrm{~mm}$, its vertical diameter is $5-8$ $\mathrm{mm}$, and its anteroposterior diameter is $10-15 \mathrm{~mm}$. The CS is composed of a network of small venous channels that may arbitrarily be divided into different compartments. The main venous influx into the CS is the superior and inferior ophthalmic veins, pterygoid plexus, and Sylvian vein. The outflow of the CS occurs via the superior and inferior petrosal sinuses. The internal carotid artery (ICA) is the most medial structure inside the CS and is contained in the so-called carotid trigone. Cranial nerves III and IV and the first and second divisions of the cranial nerve $\mathrm{V}$ (from superior to inferior) are located in the lateral dural wall of the CS (called the oculomotor trigone). Cranial nerve V courses in the central part of the CS inferolateral to the ICA. Inside, the CS is a multiseptate space, which shows intense contrast enhancement of the slower flowing venous blood. The ICA appears as a signal-intensity void structure. $^{3-7}$ Occasionally, the CS may contain fatty deposits that are normal (Fig 1). These fatty zones may be more prominent in obese patients or those with Cushing syndrome or receiving exogenous steroid therapy.

\section{Neoplastic Lesions}

\section{Schwannoma}

A trigeminal nerve schwannoma commonly involves the CS and, in $50 \%$ of instances, has a typical dumbbell-shape with bulky tumor in the Meckel cave and the prepontine cistern with a waist at the porous trigeminus. Conversely, it may be found only involving the Meckel cave (Fig 2). It may be solid or have variable cystic or hemorrhagic components with occasional fluid levels. Small tumors tend to be homogeneous, whereas large ones are frequently heterogeneous in appearance. Schwannomas are isointense-to-hypointense masses on T1 images, mostly T2 hyperintense, and show contrast enhancement. A clue to the diagnosis is that they follow the expected course of the nerves from which they arise. Schwannomas may arise from other cranial nerves in the CS, particularly cranial nerve III. Multiple CS schwannomas and bilateral acoustic ones are seen in patients with neurofibromatosis type $2 .^{9-13}$

\section{Plexiform Neurofibroma}

Plexiform neurofibromas most commonly involve the trigeminal nerve, especially its first and second branches. A suggestive imaging feature is a tortuous or fusiform enlargement of the nerves that exhibit heterogeneous signal intensity. Unlike 


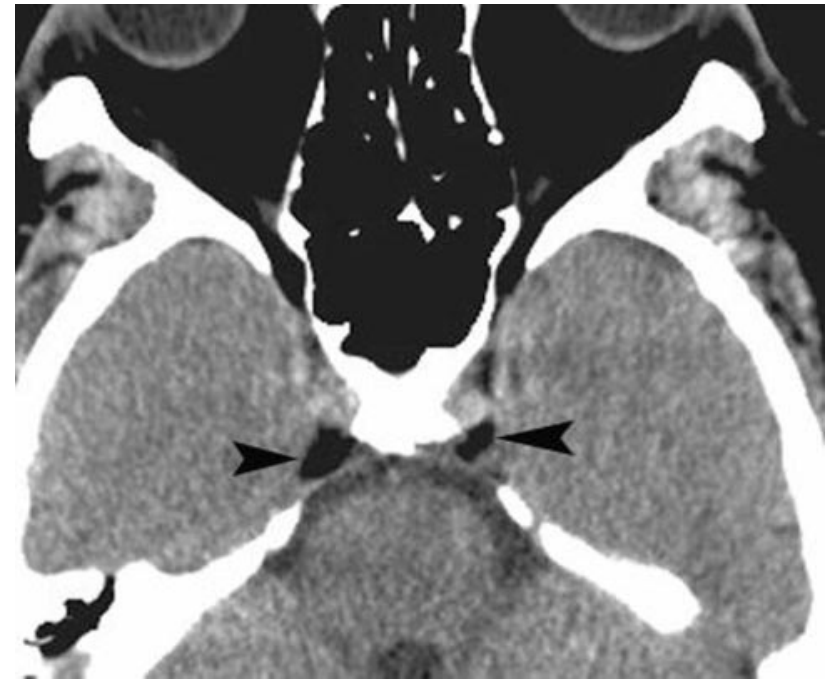

Fig 1. Normal fat deposits. Axial noncontrast CT scan shows normal and incidentally found deposits of fat (arrowheads) in the posterior CSs. These deposits may be seen in obese individuals, those taking corticosteroids, or those with Cushing syndrome. In the absence of these conditions, they have no significance.

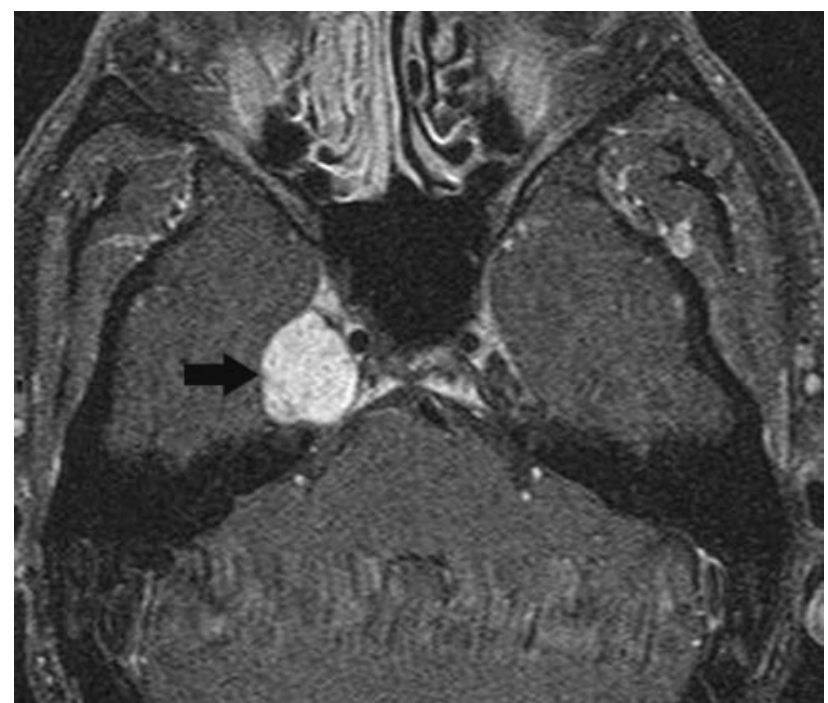

Fig 2. Schwannoma. Axial postcontrast T1-weighted image shows a well-defined enhancing mass (arrow) involving the Meckel cave on the right. Although the findings are nonspecific, the most common mass in this location is a schwannoma.

schwannomas, neurofibromas are less likely to extend to the Meckel cave. They are seen in $30 \%$ of patients with neurofibromatosis type 1 but are extremely rare outside this disease. $^{9,14}$

\section{Malignant Peripheral Nerve Sheath Tumor}

Malignant peripheral nerve sheath tumor is a high-grade sarcoma that may infiltrate the CS. Large tumor size $(>5 \mathrm{~cm})$, ill-defined infiltrative margins, rapid growth, tumor signalintensity heterogeneity, and erosion of the skull base foramina out of proportion to tumor size suggest its underlying malignant nature (Fig 3). ${ }^{9,15}$ Its imaging findings are nonspecific, and the diagnosis is made by histology.

\section{Cavernous Hemangioma}

CS hemangioma is more commonly seen during the fifth decade of life in female patients. It is among the most common

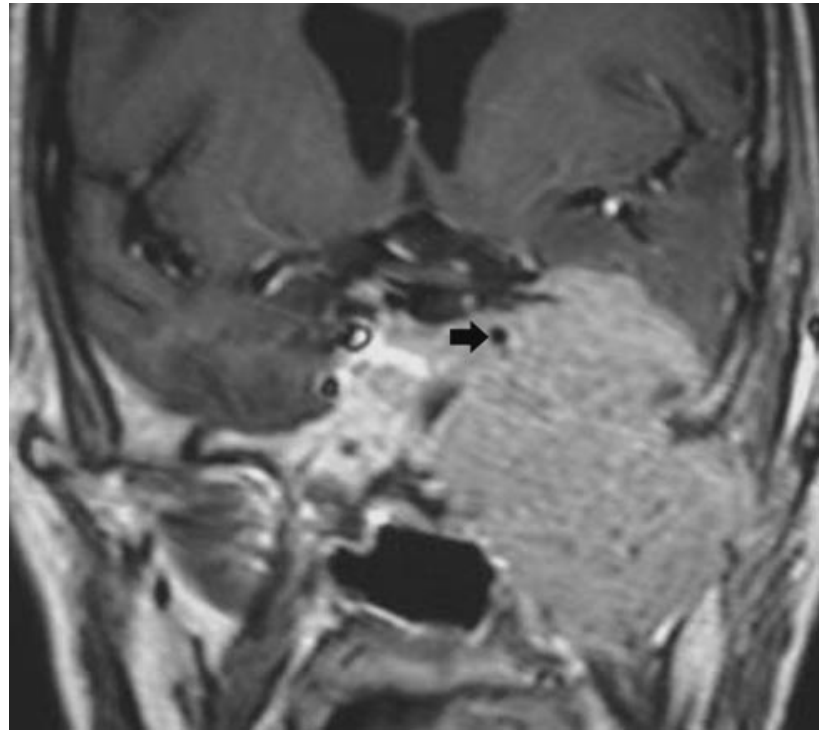

Fig 3. Malignant peripheral nerve sheath tumor. Coronal postcontrast T1-weighted image shows a large aggressive-appearing mass that involves the left CS, surrounds the ICA (arrow), erodes the middle cranial fossa floor, and extends into the infratemporal region.

primary CS tumors along with schwannoma and meningioma. This tumor is formed by sinusoidal spaces with endothelial lining that contain slow-flowing or stagnant blood. A preoperative diagnosis is important because of its propensity to bleed at the time of resection. These tumors are nearly hyperintense on T1- and T2-weighted images and are attached to the outer wall of the CS, and their diagnosis may be suggested when they show progressive "filling in" after contrast administration. Other times, they show nonspecific intense homogeneous or heterogeneous contrast enhancement (Fig 4). ${ }^{16-19}$

\section{Meningioma}

Most CS meningiomas arise from the lateral dural wall, but sometimes they may be exclusively inside the CS. A meningioma is usually hypo- to isointense with respect to gray matter in all MR imaging sequences and enhances intensely (Fig 5A). A dural tail frequently can be seen extending away from the edge of the tumor and often into the ipsilateral tentorium. Meningiomas constrict the lumen of the ICA. Meningiomas may extend inside the CS and the Meckel cave and via the porous trigeminus into the prepontine cistern. They may have an appearance very similar to schwannomas (Fig 5B). ${ }^{20-22}$

\section{Pituitary Adenoma}

Pituitary adenomas may grow laterally and invade the CS. A sign of CS invasion is encasement of the intracavernous ICA by $>30 \%$ of its diameter or tumor extension lateral to the top (12 o'clock) of the ICA. Interposition of abnormal soft tissue between the lateral wall of the CS and the ICA is a reliable indicator of CS invasion. Unlike meningiomas, pituitary adenomas generally do not narrow the ICA. ${ }^{23}$

\section{Melanocytoma}

Primary melanocytomas originate from the leptomeninges of the CS. The lesion is hyperintense with fine punctate areas of decreased signal intensity on T1-weighted images and of low signal intensity on $\mathrm{T} 2$-weighted images and shows no contrast 

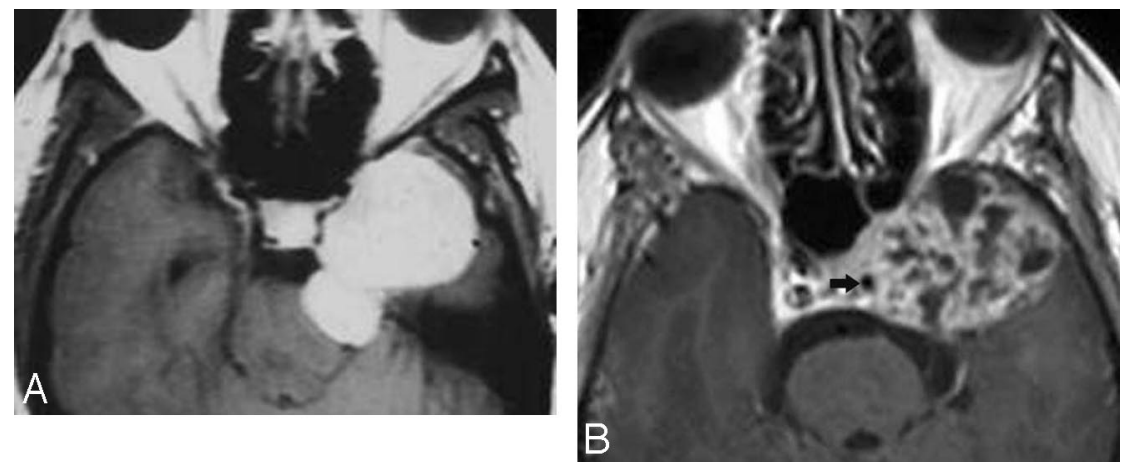

Fig 4. Cavernous hemangiomas. A, Axial postcontrast T1 weighted image shows a large and homogeneously enhancing mass arising from the lateral wall of the left CS. $B$, Axial postcontrast T1-weighted image in a different cavernoma, which shows inhomogeneous contrast enhancement but also arises from the lateral wall of the CS, pushing the ICA (arrow) medially. When a mass arises in the lateral wall of a CS, the most important differential diagnosis is that of meningioma versus cavernoma
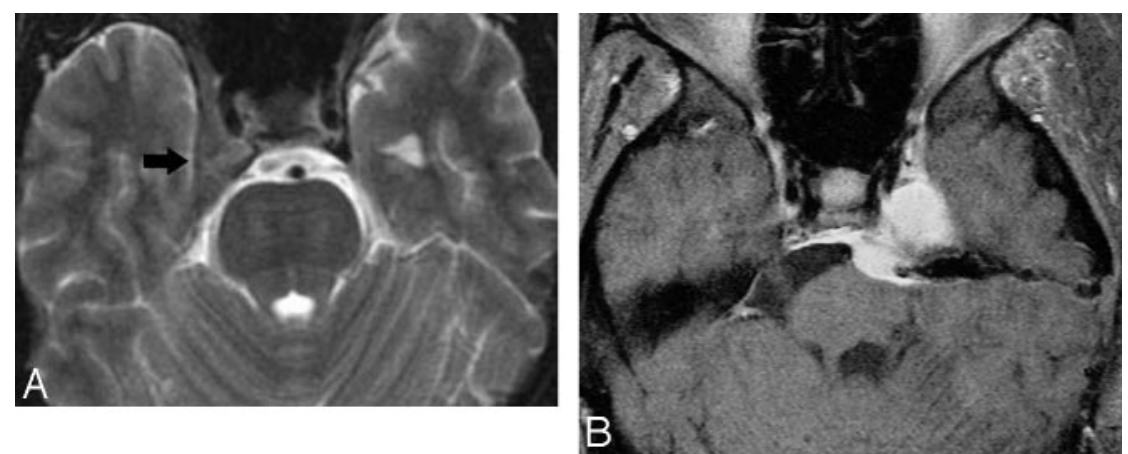

Fig 5. Meningiomas. A, Axial T2-weighted image shows a meningioma (arrow) that is isoattenuated to white matter involving the right CS and extending from the Meckel cave into the superior orbital fissure. B, Axial postcontrast T1weighted image in a different patient shows the bulk of a homogeneously enhancing mass in the Meckel cave but extending through the porous trigeminus into the prepontine cistern. A schwannoma needs to be considered in the differential diagnosis.
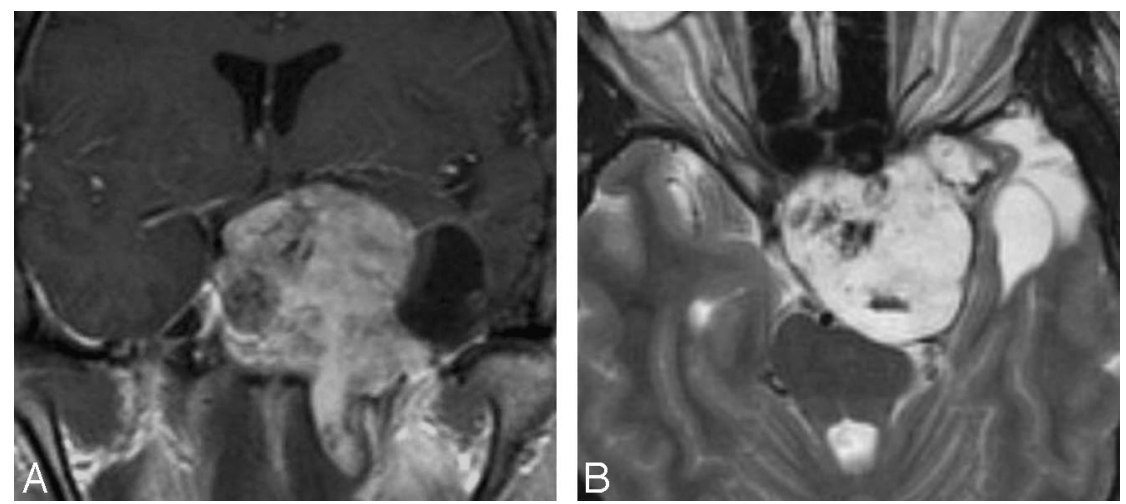

Fig 6. Chondrosarcoma. $A$, Coronal postcontrast T1-weighted image shows a large inhomogeneous-appearing mass involving the left CS, sella, suprasellar region, ipsilateral middle cranial fossa, and intratemporal region. The mass has a cystic lateral component. $B$, Axial T2-weighted image shows that the solid portion of the mass is hyperintense, a finding that is typical of chondrosarcoma

enhancement. It may be indistinguishable by imaging from primary meningeal melanoma, melanocytic schwannoma, and cavernous angioma. ${ }^{24}$

\section{Chordoma}

Chordoma is a locally invasive midline primary clival tumor that may also originate slightly more laterally from the spheno-occipital synchondrosis and may extend into the CS. On T1-weighted images, it shows intermediate signal intensity with focal high-signal-intensity areas representing hemorrhage or high protein. The tumor is of relatively high signal intensity on T2-weighted images with hypointense areas representing residual fragments or sequestrations of bone. CT shows bone destruction and calcifications. ${ }^{25,26}$

\section{Chondrosarcoma}

Chondrosarcoma may originate from the petroclival synchondrosis and infiltrate the CS. CT shows a stippled and/or amorphous calcified matrix. On MR imaging, the tumor shows variable signal intensity on T1-weighted images and characteristic high T2 signal intensity. The areas of high T1 signal intensity may reflect hemorrhage or mucin. The heterogeneous T2 signal intensity may be due to fibrocartilaginous foci or areas of chondroid mineralization. Enhancement is moderate to marked and usually heterogeneous, though occasionally it is homogeneous (Fig 6). ${ }^{26-27}$

\section{Nasopharyngeal Carcinoma}

Nasopharyngeal carcinoma is the most common primary malignant extracranial neoplasm to invade the CS. Intracranial extension may occur directly via the skull base erosion or by perineural spread along branches of the trigeminal nerve (Fig 7). Tumor can extend through the petro-occipital synchondrosis and foramen lacerum into the inferior CS or via the carotid canal to gain access to the CS without destroying bone. Once the CS is invaded, bulky masses are present in the nasopharynx. The tumor is generally hypointense to isointense (relative to muscles) on T1-weighted images and T2 hypointense and shows moderate-to-intense contrast enhancement. ${ }^{28-30}$ 


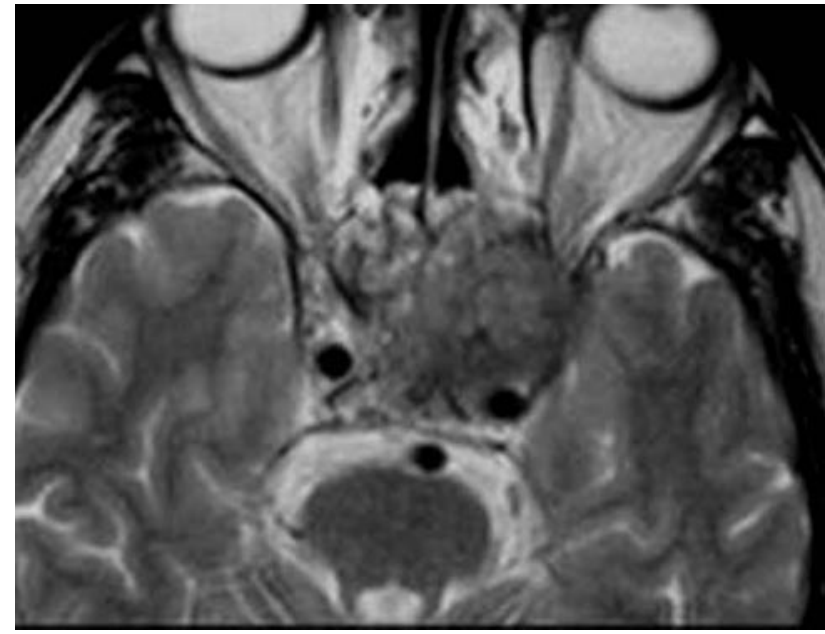

Fig 7. Nasopharyngeal squamous cell carcinoma. Axial T2-weighted image shows a relatively hypointense mass involving the left CS and sella, extending into the posterior ethmoid air cells. Invasive T2 hypointense masses are generally either neoplasias or fungal infections.

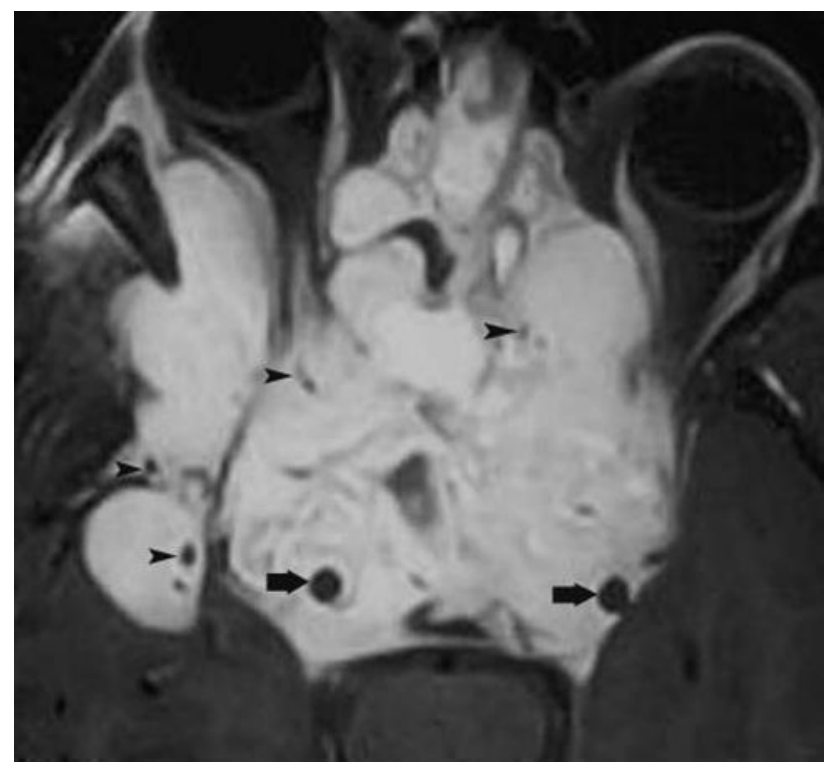

Fig 8. Juvenile angiofibroma. Axial postcontrast T1-weighted image shows a very large tumor involving both CSs and surrounding the ICAs (arrows). The mass extends into the sella, paranasal sinuses, right middle cranial fossa, and both orbits. Note flow voids (arrowheads) due to enlarged blood vessels.

\section{Juvenile Angiofibroma}

Juvenile angiofibroma is a highly vascular tumor that affects mostly adolescent boys. It can extend into the central skull base and to the anterior part of the CS through the foramen rotundum, vidian canal, or foramen lacerum. The tumor can invade the CS directly by erosion of the pterygoid bone. The characteristic signal-intensity voids on MR imaging, representing large vascular structures, are typical of this tumor (Fig 8)..$^{30-31}$

\section{Sphenoid Sinus Carcinoma}

Malignant tumors of the sphenoid sinus include squamous cell carcinoma and adenocarcinoma. They tend to destroy bone and directly spread to the CS. Sphenoid sinus carcinomas typically have low-to-intermediate T1 signal intensity and

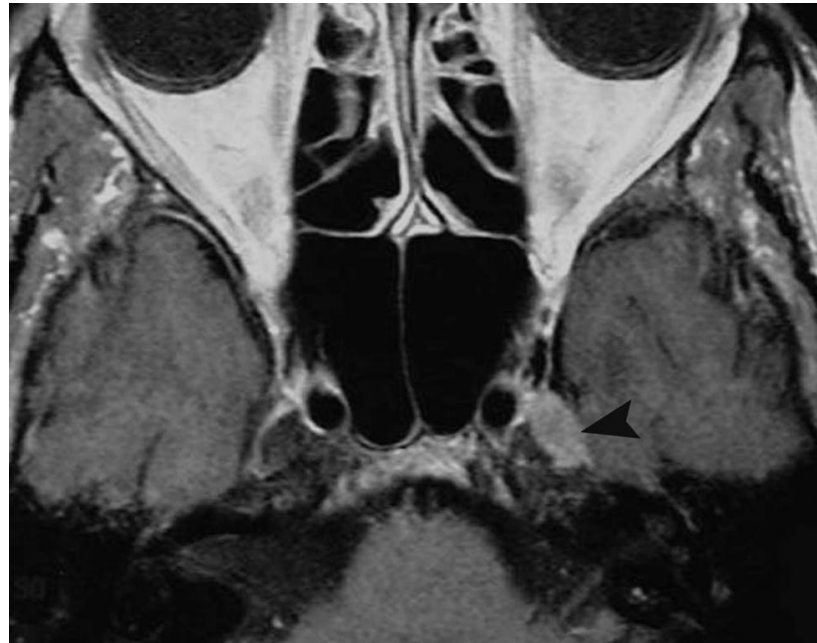

Fig 9. Metastasis. Axial postcontrast T1-weighted image shows an enhancing mass (from primary breast carcinoma) in the right the Meckel cave (arrowhead). In the absence of primary tumor elsewhere, schwannoma needs to be considered in the differential diagnosis.

low T2 signal intensity and show contrast enhancement. They have nonspecific imaging findings except that their epicenter is generally in the expected location of the sphenoid sinus. ${ }^{32-34}$

\section{Rhabdomyosarcoma}

Rhabdomyosarcoma is a mesenchymal malignant tumor occurring most often in children. Involvement of the CS is common and is a grave prognostic sign. Destruction of the skull base with intracranial and CS involvement is present in advanced cases. The signal intensity of this tumor is isointense to surrounding muscles on T1-weighted images and T2 hyperintense. The mass shows enhancement that is more than that of the surrounding muscle. ${ }^{35}$

\section{Metastases}

Metastases to the CS can be hematogenous or perineural in nature. Distant tumors with hematogenous spread to the CS are generally renal, gastric, thyroid, lung, and breast cancers. MR imaging shows CS enlargement, outward bowing of its lateral wall, and replacement of the Meckel cave with soft tissue that homogeneously enhances (Fig 9). Perineural tumor spread is commonly seen along branches of cranial nerve V. Perineural spread is most commonly seen with adenoid cystic or squamous cell carcinoma but may also be seen with lymphoma, melanoma, basal cell carcinoma, rhabdomyosarcoma, neurogenic tumors, and juvenile angiofibroma. MR imaging features of perineural tumor spread include nerve enlargement and enhancement and foraminal enlargement and destruction (Fig 10). ${ }^{36-42}$

\section{Lymphoma and Leukemia}

As with metastases, lymphoma and leukemia reach the CS by direct extension from a primary lesion or from hematogenous spread. MR imaging may show infiltrative lesions of the skull base invading the CS without arterial narrowing. Lymphoma and leukemia may also appear as diffuse enlargement and enhancement of the CS similar to the appearance of metastases. Although they tend to be T2 hypointense, most have no specific MR imaging findings, but the diagnosis may be suggested 

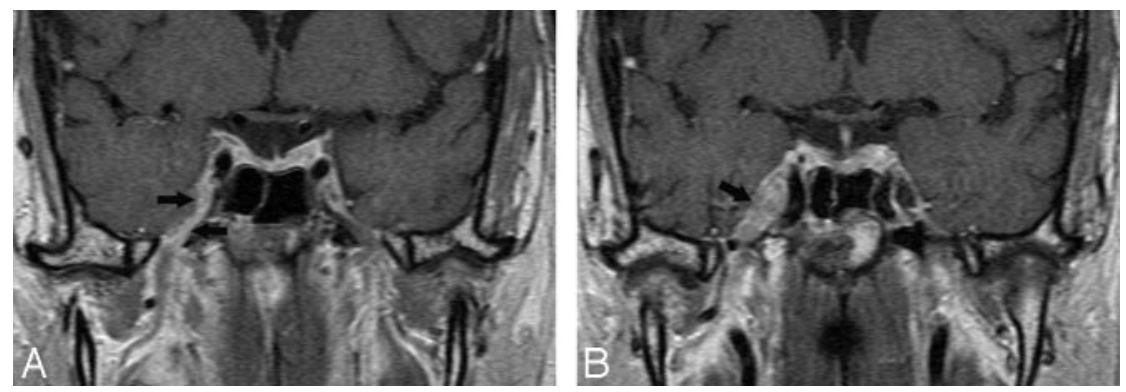

Fig 10. Perineural tumor spread. A, Coronal postcontrast T1-weighted image shows a thick enhancing third division (arrows) of the right trigeminal nerve in a patient with a small adenoid cystic carcinoma in the nasopharynx. $B$, Slightly posterior to $A$, the tumor has invaded the Gasserian ganglion and fills the Meckel cave (arrow).
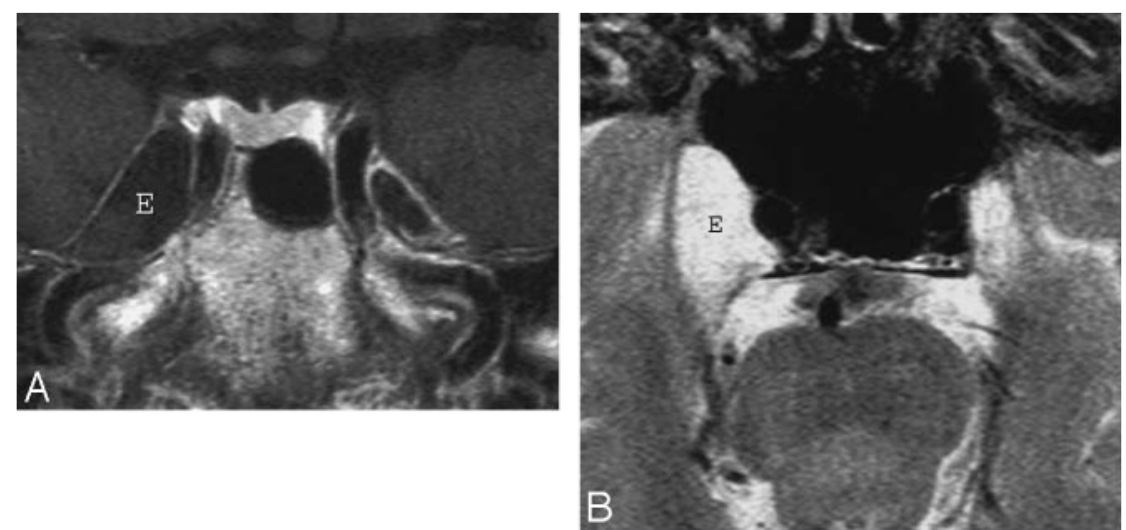

Fig 11. Epidermoid. A, Axial postcontrast T1-weighted image shows a large mass $(E)$ inside the right the Meckel cave. The mass does not enhance and is nearly isointense to CSF. $B$, Axial T2-weighted image in the same patient shows that the mass (E) is nearly as bright as CSF. Despite the large size of the lesion, clinical symptoms were mild. The differential diagnosis includes an intratemporal meningocele.

because of the clinical results. Nowadays, most patients with known lymphoma/leukemia who have been treated will have clinical evidence of relapse by the time MR imaging depicts the new lesions. ${ }^{43-45}$

\section{Posttransplantation Lymphoproliferative Disorder}

Posttransplantation lymphoproliferative disorder is a complication of organ transplantation and chronic immunosuppression that varies from B-cell hyperplasia to non-Hodgkin lymphoma. It may present as cervical lymphadenopathy, focal masses involving the Waldeyer ring, and, rarely, involvement of the CS. These lymphoid proliferations are frequently iso- to hypointense on T1- and T2-weighted images because of attenuated cellularity and being identical to lymphoma. The imaging features are nonspecific, and the diagnosis is a clinical one. $^{46}$

\section{Epidermoid and Dermoid Cysts}

An epidermoid cyst may be of extracavernous origin and extend into the CS, originate in the lateral CS wall (interdural cyst), or be a true intracavernous lesion. The mass is hypointense/isointense and T2 hyperintense. On FLAIR and highresolution T2-weighted images, it demonstrates a heterogeneous signal intensity. These cysts do not enhance. Epidermoid cysts show restricted diffusion with higher signal intensity than that of CSF on diffusion-weighted imaging (Fig 11). ${ }^{4-49}$

A dermoid cyst of the CS is usually interdural and shows smooth contours, a rounded shape, and medial displacement of the ICA without significantly narrowing it. These lesions are generally $\mathrm{T} 1$ hyperintense and $\mathrm{T} 2$ hypointense but at times may show mixed signal intensity on T2-weighted MR imaging. 50

\section{Disorders of Histiocyte Proliferation}

Langerhans cell histiocytosis is a rare disorder that may involve the skull base in pediatric patients. When it affects the skull base, it can be broadly divided into 2 forms. The more common form is confined to the petrous portion of the temporal bone, whereas the less common form may arise anywhere in the skull base and extend into the middle cranial fossa and CS. ${ }^{51-53}$

Rosai-Dorfman disease is an uncommon proliferative disorder of histiocytes of unknown etiology, which may infiltrate the CS. It may begin in the cervical lymph nodes, but extranodal sites such as the nasal cavity, orbit, and parotid gland have been reported. The disease typically manifests as dural-based enhancing masses in the cerebral convexities, suprasellar region, CSs, and petroclival regions. ${ }^{54-56}$

Erdheim-Chester disease is a rare non-Langerhans form of histiocytosis that affects multiple organs. It may infiltrate the hypothalamus, orbit, dura, and CS. It appears as dural mass lesions in the parasellar region, falx, and tentorium. The lesions enhance intensely after contrast administration. ${ }^{57,58}$

\section{Inflammatory, Infectious, and Granulomatous Lesions}

\section{Tolosa-Hunt Syndrome}

Tolosa-Hunt syndrome is a term applied to a retro-orbital pseudotumor extending to the CS. Its clinical triad includes unilateral ophthalmoplegia, cranial nerve palsies, and a dramatic response to systemic corticosteroids. The process is usually unilateral but may be bilateral (5\%). Histopathology shows a low-grade nonspecific inflammatory process with lymphocytes and plasma cells. MR imaging findings include an enlarged CS containing abnormal soft tissues that are isointense to muscle on T1-weighted images and dark or bright on 

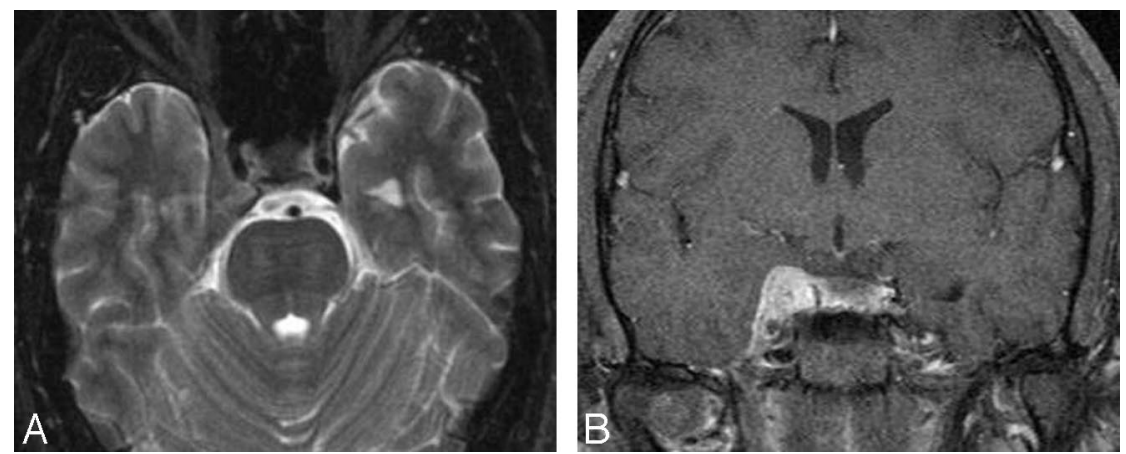

Fig 12. Tolosa-Hunt syndrome. A, Axial T2-weighted image shows hypointense soft tissue throughout the right CS and extending into the superior orbital fissure. $B$, Coronal postcontrast T1-weighted image shows that the abnormal soft tissue enhances prominently and diffusely and involves the Meckel cave.

T2-weighted images and display contrast enhancement with focal narrowing of the ICA (Fig. 12). ${ }^{59-61}$

\section{Inflammatory Myofibroblastic Tumor}

Inflammatory myofibroblastic tumor is a rare process that may affect the orbit and extend into the CS in $10 \%$ of patients. It is characterized by inflammatory cell infiltration and a variable fibrotic response. Typical MR imaging findings include focal enlargement of an extraocular muscle and a soft-tissue lesion replacing the orbital fat, which may extend into the CS. The lesions are iso- to hypointense on T2-weighted images, depending on their amount of fibrosis and cellularity, and show contrast enhancement. This tumor may be indistinguishable from idiopathic inflammatory pseudotumor by imaging (Fig 13). ${ }^{62,63}$

\section{Idiopathic Hypertrophic Pachymeningitis}

Idiopathic hypertrophic cranial pachymeningitis is a rare inflammatory disease with diffuse dural involvement that may extend to the CS. MR imaging shows enhancement and dural thickening, which may be extensive or more localized (Fig 14). When the falx and tentorium are involved, extension into the CS is relatively common. This disease may lead to CS thrombosis, white matter edema, and hydrocephalus due to venous hypertension. ${ }^{64}$

\section{Tuberculous Pachymeningitis}

Tuberculosis is, in some parts of the world, a relatively common cause of pachymeningitis that produces diffuse or focal extra-axial masses along the surfaces of the CS. The lesions enhance homogeneously and intensely and may be nodular. They are dark on T2-weighted images, and the presence of basal meningitis elsewhere may suggest the diagnosis. A sarcoid may be identical in appearance. ${ }^{65,66}$

\section{Fungal Infection}

Invasive aspergillosis may affect the sphenoid sinus in immunocompromised patients and may extend intracranially with invasion of the CS. This infection shows low signal intensity on both T1- and T2-weighted images, which is attributed to the presence of ferromagnetic elements and calcium in the fungal and mucous concretions. It exhibits intense inhomogeneous contrast enhancement (Fig 15) ${ }^{67-69}$ Rhinocerebral mucormycosis is often an acute fulminant opportunistic infection, which may affect the orbits and paranasal sinuses. MR imaging findings include enhancing soft-tissue masses in the orbital apex and CS, with thickening and lateral displacement of the

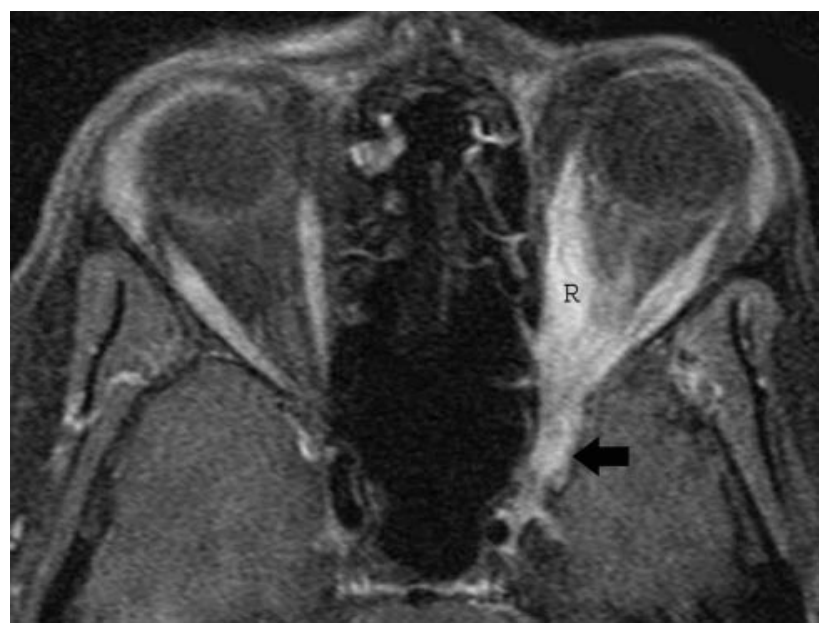

Fig 13. Inflammatory myofibroblastic tumor. A, Axial postcontrast T1-weighted image shows thickening and stranding of the left extraocular muscles more on medial rectus (R) and extension of the process into the ipsilateral CS (arrow). There is prominent enhancement of these muscles and of the retro-orbital fat.

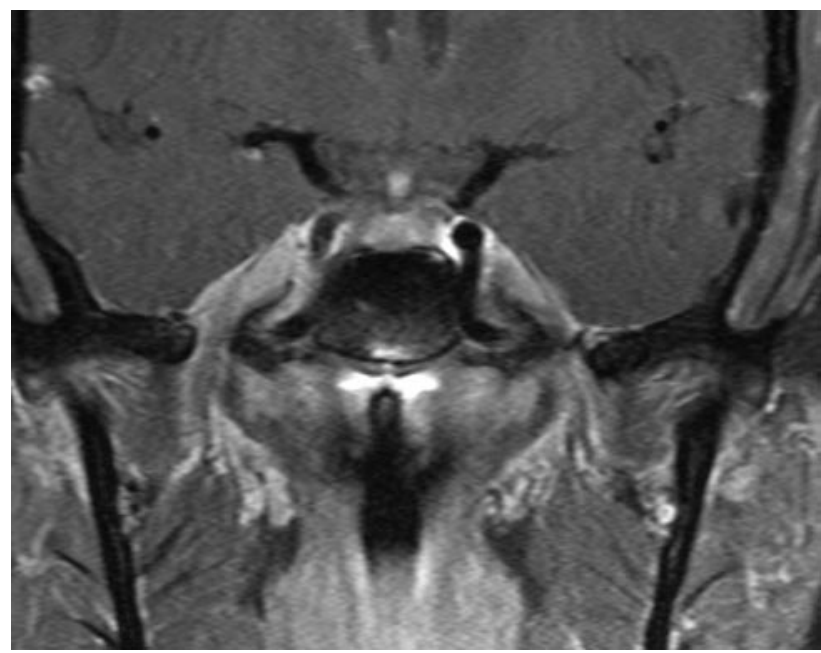

Fig 14. Idiopathic pachymeningitis. Coronal postcontrast T1-weighted image shows nonspecific thickening of the right CS at the level of the foramen ovale. There is perhaps slight thickening of the lateral dural wall of the contralateral CS

medial rectus muscle and involvement of the neighboring ethmoid sinus. ${ }^{70,71}$ Extension into the CS may result in thrombosis and thickening of the ICA walls, with narrowing of its lumen. Actinomycosis is rare and may gain access to the CS via direct extension from the ear or sinuses or by hematogenous spread. Its MR imaging features are nonspecific. ${ }^{72,73}$ These 

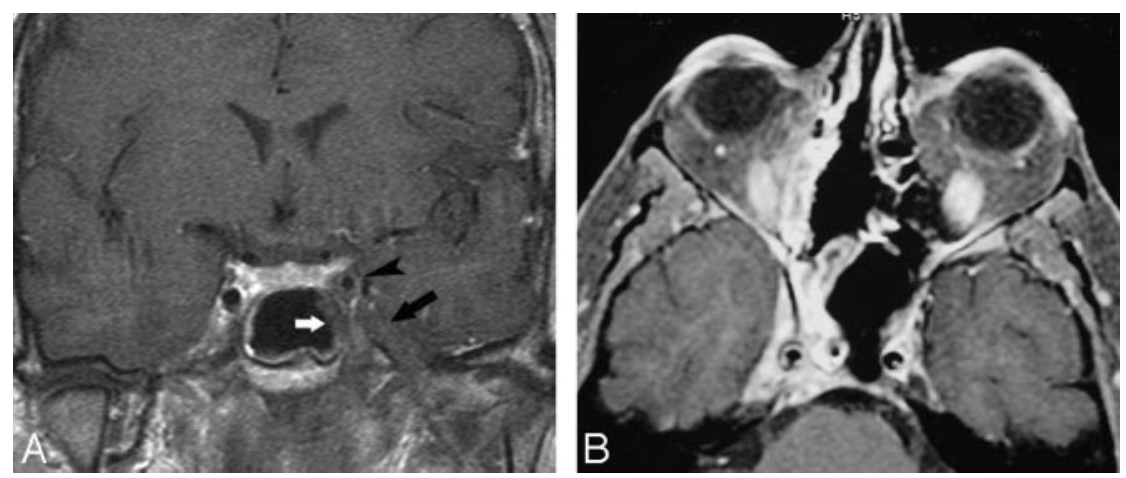

Fig 15. Fungal infection. $A$, Coronal postcontrast T1-weighted image shows involvement by aspergillosis of the mucosa in the left sphenoid sinus (white arrow), which extends laterally and has resulted in thrombosis of the adjacent CS (black arrow). The intracavernous ICA (arrowhead) is narrowed, and its walls are significantly thickened. $B$, In a different patient, an axial postcontrast T1-weighted image shows enhancing mucosa in the right sphenoid sinus with enhancing soft tissues in the ipsilateral CS (including the Meckel cave), with narrowing of the ICA and significant thickening of its walls.

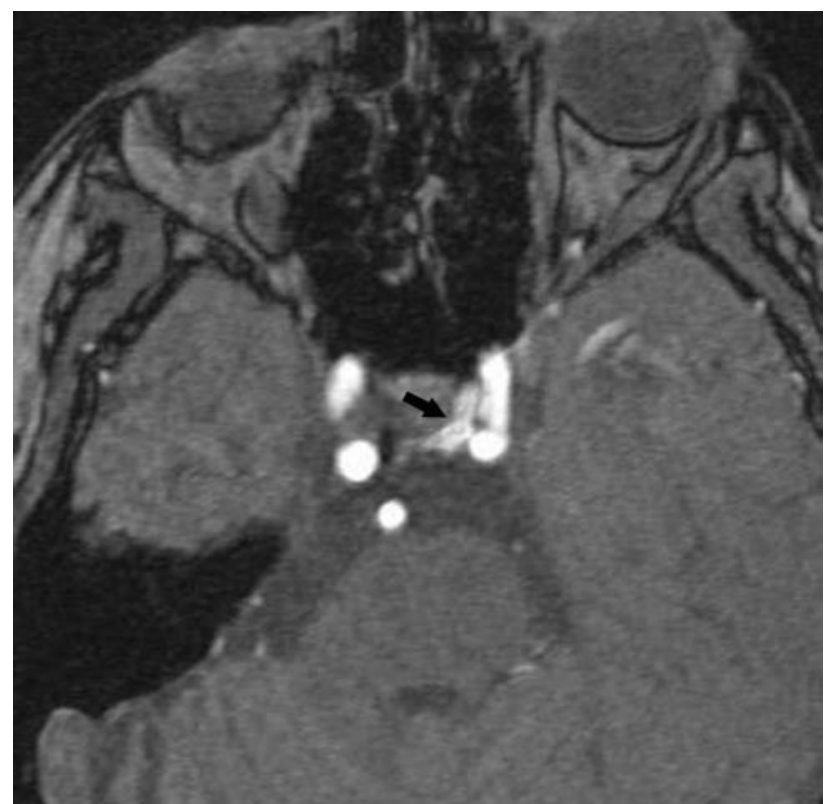

Fig 16. ICA to a CS fistula. Axial source image from an MR angiogram shows flow-related enhancement in the medial (arrow) left CS from a direct-type fistula.

infections should always be considered in immunosuppressed patients who present with any type of paranasal sinus disease that extends beyond the bony walls of that sinus.

\section{Wegener Granulomatosis}

Wegener granulomatosis is a rare disorder of unknown etiology associated with necrotizing granulomas in the paranasal sinuses, nasal cavities, and/or orbits. CS involvement is rare. MR imaging shows meningeal thickening and enhancement that may extend to the CS. The CS shows low T2 signal intensity due to presence of fibrous tissues and intense and homogeneous contrast enhancement. ${ }^{74-76}$ In most patients, the diagnosis has been established before any imaging studies are obtained.

\section{Sarcoidosis}

Dural involvement can occur with sarcoidosis and extend to the CS. The presence of multiple dural-based lesions, pachymeningitis or leptomeningeal enhancement, thickened cranial nerves, thickened hypothalamus, multiple scattered brain lesions, and periventricular multifocal white matter lesions should raise the possibility of the diagnosis of sarcoidosis. ${ }^{77,78}$ The appearance of the lesions is nonspecific, but they tend to be dark on T2-weighted images and may be accompanied by neighboring cerebral edema.

\section{Vascular Lesions}

\section{Carotid Cavernous Fistula}

Carotid cavernous fistula (CCF) is an abnormal connection between the carotid arterial system and the CS, which may be classified into 4 types. Direct CCF (type A) is a high-flow communication between the ICA and the CS that occurs after trauma or secondary to a ruptured aneurysm of the cavernous ICA. These lesions present acutely with pulsating exophthalmos, chemosis, and CS syndrome. Dural CCFs (types B-D) are low-flow fistulas occurring between meningeal branches of the carotid artery and CS, which tend to have milder symptoms than direct fistulas. MR imaging findings of CCFs include a dilated CS with multiple signal-intensity void structures that are associated with proptosis and an enlarged superior ophthalmic vein. ${ }^{79-82}$ On gradient-echo images, these flow voids shows high signal intensity. The presence of flowrelated enhancement in the CS on MR angiography suggests the diagnosis in the right clinical setting. Other supporting findings are a "dirty" appearance of the retro-orbital fat and enlargement of the extraocular muscles. Due to the presence of intracavernous communications, very high-flow fistulas may result in enlargement of both CSs (Fig 16).

\section{Aneurysms}

Cavernous carotid aneurysms compose $5 \%$ of giant aneurysms $(>2.5 \mathrm{~cm}$ in diameter). They may produce CS syndrome by virtue of mass effect, inflammation, or rupture into the CS, with subsequent development of a CCF. Most are idiopathic, but they may occasionally be traumatic or mycotic in nature. A patent aneurysm shows signal-intensity void on spin-echo MR imaging sequences. Partially thrombosed giant aneurysms show mixed signal intensities representing various stages of clot in their walls (due to chronic dissections) or within their lumen. Flowing blood through the patent portion of the lumen appears as a signal-intensity void on spin-echo images and high signal intensity on gradient techniques (Fig 17). ${ }^{83-86}$

\section{Thrombosis}

CS thrombosis may be secondary to infection of the sinonasal cavities, orbits, and/or the middle third of the face. MR imaging signs of CS thrombosis include changes in signal intensity and/or in the size and contour of the CS. Although subacute 

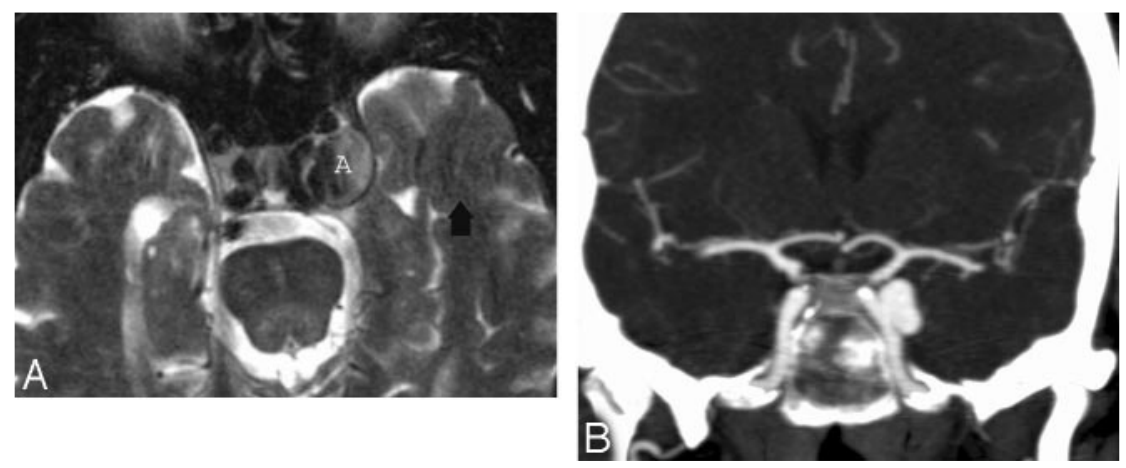

Fig 17. Intracavernous aneurysms. A, Axial T2-weighted image shows a left intracavernous ICA (A) aneurysm. Note flow artifacts (arrow) confirming the pulsatile nature of the lesions. $B$, Coronal postcontrast maximum-intensity image from a CT angiogram in the same patient shows the left intracavernous aneurysm.
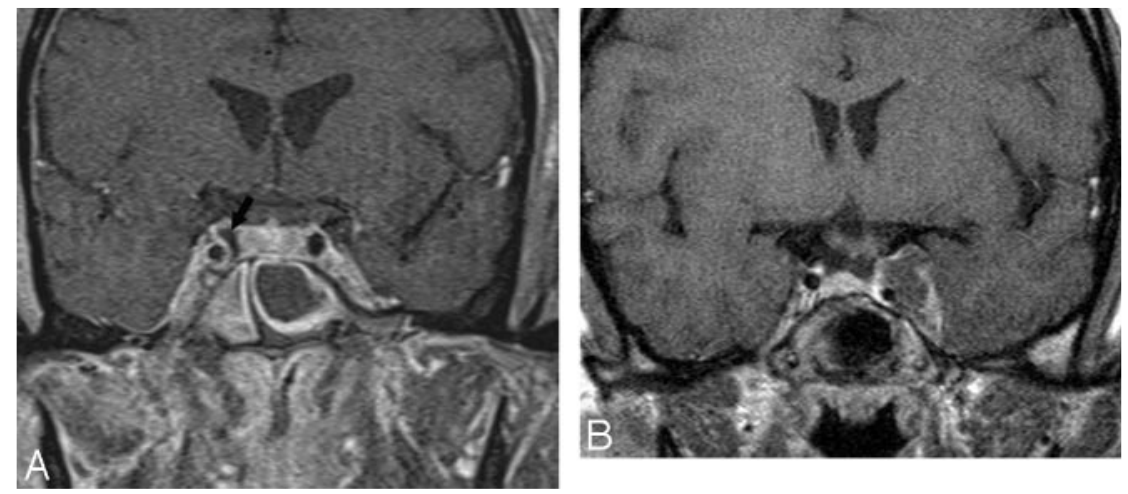

Fig 18. CS thrombosis. $A$, Coronal postcontrast T1-weighted image shows an enlarged and inhomogeneous-appearing right CS that contains areas of low signal intensity (arrow) compatible with clot. $B$, Coronal postcontrast T1-weighted image in a different patient shows a large nonenhancing clot expanding the left CS. The ipsilateral ICA is slightly narrowed.

thrombus exhibits high signal intensity on all pulse sequences and is easy to recognize, acute thrombosis may be isointense and difficult to diagnose. Enhancement of the peripheral margins of an enlarged CS may suggest a clot within it. Indirect signs that help to suggest the diagnosis are dilation of the superior ophthalmic veins, exophthalmos, and increased dural enhancement along the lateral border of CS and ipsilateral tentorium (Fig 18). ${ }^{87-89}$ The presence of sinusitis and appropriate clinical symptoms confirm the diagnosis.

\section{References}

1. Boardman JF, Rothfus WE, Dulai HS. Lesions and pseudolesions of the cavernous sinus and petrous apex. Otolaryngol Clin North Am 2008;41:195-213

2. Lee JH, Lee HK, Park JK, et al. Cavernous sinus syndrome: clinical features and differential diagnosis with MR imaging. AJR Am J Roentgenol 2003;181:583-90

3. Castillo M. Imaging of the upper cranial nerves I, III-VIII, and the cavernous sinuses. Magn Reson Imaging Clin North Am 2002;10:415-31

4. Larson TL. Petrous apex and cavernous sinus: anatomy and pathology. Semin Ultrasound CT MR 1993;14:232-46

5. Ruscalleda J. Imaging of parasellar lesions. Eur Radiol 2005;15:549-59

6. Korogi Y, Takahashi M, Sakamoto Y, et al. Cavernous sinus: correlation between anatomic and dynamic gadolinium-enhanced MR imaging findings. Radiology 1991;180:235-37

7. Yagi A, Sato N, Taketomi A, et al. Normal cranial nerves in the cavernous sinuses: contrast-enhanced three-dimensional constructive interference in steady state MR imaging. AJNR Am J Neuroradiol 2005;26:946-50

8. Eisenberg MB, Al-Mefty O, DeMonte F, et al. Benign nonmeningeal tumors of the cavernous sinus. Neurosurgery 1999;44:949-55

9. Weber A, Motandon C, Robson C. Neurogenic tumors of the neck. Radiol Clin North Am 2000;39:1077-90

10. Majoie C, Hulsmans F, Castelijns J, et al. Primary nerve-sheath tumors of the trigeminal nerve: clinical and MR findings. Neuroradiology 1999;41:100-08

11. Catalano P, Fang-Hui E, Som P. Fluid-fluid levels in benign neurogenic tumors. AJNR Am J Neuroradiol 1997;18:385-87

12. Yamada K, Ohta T, Miyamoto T. Bilateral trigeminal schwannomas associated with von Recklinghausen disease. AJNR Am J Neuroradiol 1992;13:299-300

13. Lo $P$, Harper $C$. Intracavernous schwannoma of the abducens nerve: a review of the clinical features, radiology and pathology of an unusual case. J Clin Neurosci 2001;8:357-60

14. Gholkar A, Stack J, Isherwood I. Case report: plexiform trigeminal neurofibroma. Clin Radiol 1988;39:313-15

15. Lee JH, Lee HK, Choi CG, et al. Malignant peripheral nerve sheath tumor in the parapharyngeal space: tumor spread through the eustachian tube. $A J N R$ Am J Neuroradiol 2001;22:748-50

16. Tannouri F, Divano L, Caucheteur V. Cavernous hemangioma of the cavernous sinus: case report and review of literature. Neuroradiology 2001;43:317-20

17. Sohn CH, Kim SP, Kim IM, et al. Characteristic MR imaging findings of cavernous hemangiomas in the cavernous sinus. AJNR Am J Neuroradiol 2003;24:1148-51

18. Salanitri GC, Stuckey SL, Murphy M. Extracerebral cavernous hemangioma of the cavernous sinus: diagnosis with MR imaging and labeled red cell blood pool scintigraphy. AJNR Am J Neuroradiol 2004;25:280-84

19. Jinhu Y, Jianping D, Xin L, et al. Dynamic enhancement features of cavernous sinus cavernous hemangiomas on conventional contrast-enhanced MR imaging. AJNR Am J Neuroradiol 2008;29:577-81

20. Vieira J, Cukiert A, Liberman B. Evaluation of magnetic resonance imaging criteria for cavernous sinus invasion in patients with pituitary adenomas: logistic regression analysis and correlation with surgical findings. Surgical Neurol 2006;65:130-35

21. Cottier J, Destrieux C, Brunereau L, et al. Cavernous sinus invasion by pituitary adenoma: MR imaging. Radiology 2000;215:463-69

22. Majós C, Coll S, Aguilera C, et al. Imaging of giant pituitary adenomas. Neuroradiology 1998;40:651-55

23. Hirsch WL, Sekhar LN, Lanzino G, et al. Meningiomas involving the cavernous sinus: value of imaging for predicting surgical complications. AJR Am J Roentgenol 1993;60:1083-88

24. Faro S, Koenigsberg R, Turtz A, et al. Melanocytoma of the cavernous sinus: CT and MR findings. AJNR Am J Neuroradiol 1996;17:1087-90

25. Erdem E, Angtuaco E, Hemert R, et al. Comprehensive review of intracrania chordoma. Radiographics 2003;23:995-1009

26. Lanzino G, Sekhar L, Hirsch W, et al. Chordomas and chondrosarcomas involving the cavernous sinus: review of surgical treatment and outcome in 31 patients. Surg Neurol 1993;40:359-71

27. Meyers S, HirschV, Curtin H, et al. Chondrosarcomas of the skull base: MR imaging features. Radiology 1992;84:103-08

28. Dubrulle F, Souillard R, Hermans R. Extension patterns of nasopharyngeal carcinoma. Eur Radiol 2007;17:2622-30

29. Chin S, Fatterpekar G, Chen C, et al. MR imaging of diverse manifestations of nasopharyngeal carcinomas. AJR Am J Roentgenol 2003;180:1715-22

30. Chong VF, Khoo JB, Fan YF. Imaging of the nasopharynx and skull base. Neuroimaging Clin N Am 2004;14:695-719

31. Schick B, Kahle G. Radiological finding in angiofibroma. Acta Radiologica 2000;41:585-93

32. Ishida H, Mohri M, Amatsu M. Invasion of the skull base by carcinomas: histopathologically evidenced findings with CT and MRI. Eur Arch Otorhinolaryngol 2002;259:535-39

33. Maroldi R, Ravanelli M, Borghesi A, et al. Paranasal sinus imaging. Eur J Radio 2008;66:372-86 
34. Loevner LA, Sonners AI. Imaging of neoplasms of the paranasal sinuses. $\mathrm{Neu}$ roimaging Clin North Am 2004;14:625-46

35. Lee J, Lee M, Lee B, et al. Rhabdomyosarcoma of the head and neck in adults: MR and CT findings. AJNR Am J Neuroradiol 1996;17:1923-28

36. Caldemeyer K, Mathews V, Righi $\mathrm{P}$, et al. Imaging features and clinical significance of perineural spread or extension of head and neck tumors. Radiograhics 1998;18:97-110

37. Williams LS. Advanced concepts in the imaging of perineural spread of tumor to the trigeminal nerve. Top Magn Reson Imaging 1999;10:376-83

38. Nemzek W, Hecht S, Gandour-Edwards R, et al. Perineural spread of head and neck tumors: how accurate is MR imaging? AJNR Am J Neuroradiol 1998;19:701-06

39. Chang PC, Fischbein NJ, McCalmont TH, et al. Perineural spread of malignant melanoma of the head and neck: clinical and imaging features. AJNR Am J Neuroradiol 2004;25:5-11

40. Laccourreye O, Bély N, Halimi P, et al. Cavernous sinus involvement from recurrent adenoid cystic carcinoma. Ann Otol Rhinol Laryngol 1994; 103:822-25

41. Takami T, Ohata K, Tsuyuguchi N, et al. Cavernous sinus metastasis from thyroid papillary adenocarcinoma. J Clin Neurosci 2002;9:598-600

42. Sharkawi E, Tumuluri K, Olver JM. Metastatic choriocarcinoma causing cavernous sinus syndrome. Br J Ophthalmol 2006;90:654-55

43. Heisman T, Tschirch F, Schneider J, et al. Burkitt's lymphoma with bilateral cavernous sinus and mediastinal involvement in a child. Pediatr Radiol 2003;33:719-21

44. Kalina P, Black K, Woldenberg R. Burkitt's lymphoma of the skull base presenting as cavernous sinus syndrome in early childhood. Pediatr Radiol 1996;26:416-17

45. Karadag D, Karagulle A, Erden I, et al. Trigeminal nerve involvement in T-cell acute lymphoblastic leukemia: value of MR imaging. Eur J Radiol 2002;44:16-18

46. Gordon A, Loevner L, Sonners A, et al. Posttransplantation lymphoproliferative disorder of the paranasal sinuses mimicking invasive fungal sinusitis: case report. AJNR Am J Neuroradiol 2002;23:855-57

47. Bonde V, Goel A. Interdural cavernous sinus epidermoid cyst. J Clin Neurosci 2008;15:212-14

48. Tatagiba M, Iaconetta G, Samii M. Epidermoid cyst of the cavernous sinus: clinical features, pathogenesis and treatment. Br J Neurosurg 2000;14:571-75

49. Chen S, Ikawa F, Kurisu K, et al. Quantitative MR evaluation of intracranial epidermoid tumors by fast fluid-attenuated inversion recovery imaging and echoplanar diffusion-weighted imaging. AJNR Am J Neuroradiol 2001;22: 1089-96

50. Tun K, Celikmez RC, Okutan O, et al. Dermoid tumour of the lateral wall of the cavernous sinus. J Clin Neurosci 2008;15:820-23. Epub 2008 May 6

51. Schmidt S, Eich G, Hanquinet S, et al. Extra-osseous involvement of Langerhans' cell histiocytosis in children. Pediatr Radiol 2004;34:313-21. Epub 2004 Jan 23

52. Hurley M, Meara A, Fogarty E, et al. Langerhans' cell histiocytosis of the clivus: case report and literature review. Pediatr Radiol 2004;34:267-70

53. Krishna H, Behari S, Pal L, et al. Solitary Langerhans-cell histiocytosis of the clivus and sphenoid sinus with parasellar and petrous extensions: case report and a review of literature. Surg Neurol 2004;62:447-54

54. Wang E, Anzai Y, Paulino A. Rosai-Dorfmann disease presenting with isolated bilateral orbital masses: report of two cases. AJNR Am J Neuroradiol 2001;22:1386-88

55. Kattner KA, Stroink AR, Roth TC, et al. Rosai-Dorfman disease mimicking parasagittal meningioma: case presentation and review of literature. Surg Neurol 2000;53:452-57

56. Udono H, Fukuyama K, Okamoto H, et al. Rosai-Dorfman disease presenting multiple intracranial lesions with unique findings on magnetic resonance imaging. J Neurosurg 1999;91:335-39

57. Caparros-Lefebvre D, Pruvo JP, Remy M, et al. Neurologic aspects of ErdheimChester disease. AJNR Am J Neuroradiol 1995;16:735-40

58. Johnson M, Aulino J, Jagasia M, et al. Erdheim-Chester disease mimicking multiple meningiomas syndrome. AJNR Am J Neuroradiol 2004;25:134-37

59. Cakirer S. MRI findings in the patients with the presumptive clinical diagnosis of Tolosa-Hunt syndrome. Eur Radiol 2003;13:17-28. Epub 2002 Jun 4

60. Calistri V, Mostardini C, Pantano P, et al. Tolosa-Hunt syndrome in a patient with systemic lupus erythematosus. Eur Radiol 2002;12:341-44. Epub $2001 \mathrm{Jul}$ 12
61. de Arcaya A, Cerezal L, Canga A, et al. Neuroimaging diagnosis of Tolosa-Hunt syndrome: MRI contribution. Headache 1999;39:321-25

62. Mahr MA, Salomao DR, Garrity GA. Inflammatory orbital pseudotumor with extension beyond the orbit. Am J Ophthalmol 2004;138:396-400

63. Bencherif B, Zouaoui A, Chedid G, et al. Intracranial extension of an idiopathic orbital inflammatory pseudotumor. AJNR Am J Neuroradiol 1993;14:181-84

64. Lee Y, Chueng Y, Hsu S, et al. Idiopathic hypertrophic cranial pachymeningitis: case report with 7 years of imaging follow-up. AJNR Am J Neuroradiol 2003;24:119-23

65. Morgado C, Ruivo N. Imaging meningo-encephalic tuberculosis. Eur J Radiol 2005;55:188-92

66. Wilson JD, Castillo M. Magnetic resonance imaging of granulomatous inflammations: sarcoidosis and tuberculosis. Top Magn Reson Imaging 1994;6:32-40

67. Aribandi M, McCoy V, Bazan C. Imaging features of invasive and noninvasive fungal sinusitis: a review. Radiographics 2007;27:1283-96

68. Parikh SL, Venkatraman G, DelGaudio JM. Invasive fungal sinusitis: a 15-year review from a single institution. Am J Rhinol 2004;18:75-81

69. Stringer SP, Ryan MW. Chronic invasive fungal rhinosinusitis. Otolaryngol Clin North Am 2000;33:375-87

70. Chan LL, Singh S, Jones D, et al. Imaging of mucormycosis skull base osteomyelitis. AJNR Am J Neuroradiol 2000;21:828-31

71. Mandava $\mathrm{P}$, Chaljub G, Patterson K, et al. MR imaging of cavernous sinus invasion by mucormycosis: a case study. Clin Neurol Neurosurg 2001;103: 101-04

72. Ohta S, Nishizawa S, Namba H, et al. Bilateral cavernous sinus actinomycosis resulting in painful ophthalmoplegia: case report. J Neurosurg 2002;96: $600-02$

73. Pagliani L, Campi L, Cavallini GM. Orbital actinomycosis associated with painful ophthalmoplegia: actinomycosis of the orbit. Ophthalmologica 2006;220:201-05

74. Goldberg AL, Tievsky AL, Jamshidi S. Wegener's granulomatosis invading the cavernous sinus: a CT demonstration. J Comput Assist Tomogr 1983;7:701-03

75. Provenzale JM, Mukherii S, Allen NB, et al. Orbital involvement by Wegener's granulomatosis: imaging findings. AJR Am J Roentgenol 1996;166:929-34

76. Provenzale JM, Allen NB. Wegener granulomatosis: CT and MR findings. AJNR Am J Neuroradiol 1996;17:785-92

77. Sandhu FA, Schellinger D, Martuza RL. A vascular sarcoid mass mimicking a convexity meningioma. Neuroradiology 2000;42:195-98

78. Koyama $\mathrm{T}$, Ueda $\mathrm{H}$, Togashi $\mathrm{K}$, et al. Radiologic manifestations of sarcoidosis in various organs. Radiographics 2004;24:87-104

79. Barrow DL, Spector RH, Braun IF, et al. Classification and treatment of spontaneous carotid-cavernous sinus fistulas. J Neurosurg 1985;62:248-56

80. Gupta A, Purkayastha S, Krishnamoorthy T, et al. Endovascular treatment of direct carotid cavernous fistulae: a pictorial review. Neuroradiology 2006;48:831-39

81. Kirsch M, Henkes H, Liebig T. Endovascular management of dural carotidcavernous sinus fistulas in 141 patients. Neuroradiology 2006;48:486-90

82. Hirai T, Korogi Y, Hamatake S, et al. Three-dimensional FISP imaging in the evaluation of carotid cavernous fistula: comparison with contrast-enhanced CT and spin-echo MR. AJNR Am J Neuroradiol 1998;19:253-59

83. Rubinstein D, Sandberg EJ, Breeze RE, et al. T2-weighted three-dimensional turbo spin-echo MR of intracranial aneurysms. AJNR Am J Neuroradiol 1997; 18:1939-43

84. Wilms G, Demaerel P, Bosmans $\mathrm{H}$, et al. MRI of non-ischemic vascular disease: aneurysms and vascular malformations. Eur Radiol 1999;9:1055-60

85. White PM, Wardlaw JM, Easton V. Can noninvasive imaging accurately depict intracranial aneurysms? A systematic review. Radiology 2000;217: 361-70

86. Atlas SW, Grossman RI, Goldberg HI, et al. Partially thrombosed giant intracranial aneurysms: correlation of MR and pathologic findings. Radiology 1987;162:111-14

87. Schuknecht B, Simmen D, Yuksel C, et al. Tributary venosinus occlusion and septic cavernous thrombosis: CT and MR findings. AJNR Am J Neuroradiol 1998;19:617-26

88. Chen J, Mukherjee P, Dillon W, et al. Restricted diffusion in bilateral optic nerves and retinas as an indicator of venous ischemia caused by cavernous sinus thrombophlebitis. AJNR Am J Neuroradiol 2006;27:1815-16.

89. Wang A-G, Liu J-H, Shyong M-P, et al. Magnetic resonance imaging in chronic cavernous sinus thrombosis. Neuroophthalmology 2000;23:29-34 